\title{
AVALIAÇÃO DE ACESSIBILIDADE FÍSICA EM UNIVERSIDADES FEDERAIS NO BRASIL: UMA REVISÃO SISTEMÁTICA DA LITERATURA
}

\section{EVALUATION OF PHYSICALACCESSIBILITY IN FEDERAL UNIVERSITIES IN BRAZIL: A SYSTEMATIC REVIEW OF LITERATURE}

\begin{abstract}
Ana Tereza de Assis Santos ${ }^{1}$, Esp.
Maiana Cunha Araújo², M.Sc.

Vilma Maria Villarouro², D.Sc.
\end{abstract}

(1) UFPE

e-mail: tereza.arquitetura@gmail.com

(2) UFPE

e-mail: maianacunha.arq@gmail.com

(3) UFPE

e-mail: villarouco@hotmail.com

Acessibilidade, revisão sistemática, universidades

Este artigo tem por objetivo identificar a produção acadêmica relacionada à avaliação de acessibilidade física em universidades no período entre 2007 e 2017. Para isto foi feita uma revisão sistemática de literatura por dois pesquisadores. A busca foi realizada no portal de periódicos da CAPES, onde foram selecionados os critérios de inclusão e exclusão para a escolha dos trabalhos.

Accessibility, systematic review, universities.

This article aims to identify the academic production related to the evaluation of physical accessibility in universities in the period between 2007 and 2017. For this, a systematic review of literature was done by two researchers. The search was conducted on the portal of CAPES, where the inclusion and exclusion criteria for the selection of works were selected. 


\section{$16^{\circ}$ \\ ERGODESIGN USIHC CINAHPA}

\section{Introdução}

As universidades são espaços públicos de grande importância, e devem ter como característica uma infraestrutura física acessível a todos os públicos. Os estudos sobre acessibilidade têm a inclusão como principal motivador, e têm se desenvolvido em aspectos técnicos, tecnológicos e científicos, visando a equivalência de oportunidades. Sob este aspecto, a acessibilidade física pode ser vista como um ponto de absoluta necessidade, principalmente em ambientes acadêmicos.

De acordo com o decreto 5.296/2004, acessibilidade é a "condição para utilização, com segurança e autonomia, total ou assistida, dos espaços, mobiliários e equipamentos urbanos, das edificações, dos serviços de transporte e dos dispositivos, sistemas e meios de comunicação e informação, por pessoa portadora de deficiência ou com mobilidade reduzida". Eventos bastante representativos já discutiram os problemas das escolas não acessíveis, como a Conferência Mundial de Necessidades Educativas Especiais: Acesso e Qualidade, realizada pela UNESCO em 1994.

Um evento também representativo e com efetiva participação do Brasil foi a Convenção sobre os direitos da pessoa com deficiência, onde os participantes assumiram o compromisso de assegurar um sistema de educação inclusivo em todos os níveis de ensino. [GOMES et al, 2011]

No Brasil, esta ação pode ser identificada através do programa Incluir, cumprindo o que consta nos decretos $\mathrm{n}^{0} 5.296 / 2004 \mathrm{e} \mathrm{n}^{\circ}$ 5.626/2005 e no edital INCLUIR de 04/2008. Além disso, a lei 13.409/2016 determina que pessoas com deficiência sejam aptas para $\mathrm{o}$ sistema de cotas já existente para outras categorias, e a lei que instituiu esse sistema [lei 12.711/2012] acrescenta que a inclusão de pessoas neste sistema tenha representatividade proporcional à existente na população brasileira, $16^{\circ}$ Ergodesign - Congresso Internacional de Ergonomia e Usabilidade de Interfaces Humano Tecnológica: Produto, Informações Ambientes Construídos e Transporte

$16^{\circ}$ USIHC - Congresso Internacional de Ergonomia e Usabilidade de Interfaces Humano Computador

CINAHPA | 2017 - Congresso Internacional de Ambientes Hipermídia para Aprendizagem. seguindo os dados do último senso do IBGE disponível.

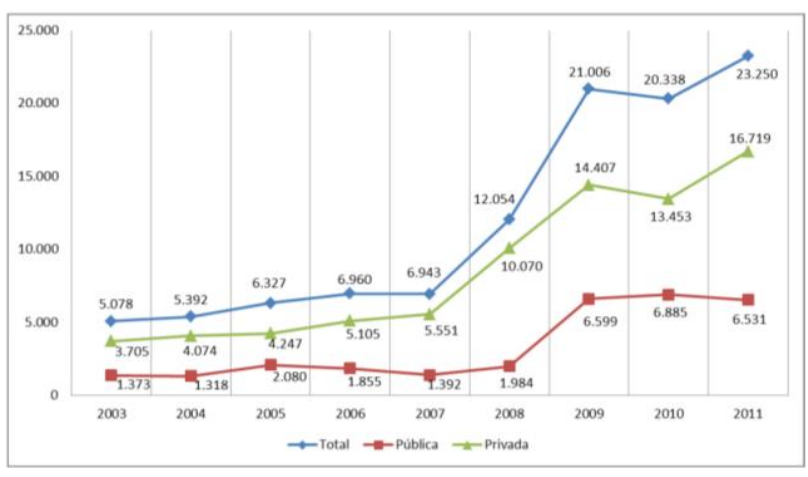

Figura 1: Evolução das matrículas de estudantes com deficiência na educação superior - Fonte: Documento orientador do programa Incluir - Acessibilidade na Educação Superior SECADI/SESu-2013

Estas, entre outras ações, demonstram a necessidade urgente de adaptação dos espaços físicos das universidades, portanto este artigo visa fazer uma revisão sistemática acerca dos estudos sobre acessibilidade física em universidades brasileiras, buscando conteúdo com maior representatividade e maior acessibilidade no meio acadêmico. Para tanto foram utilizados como base de pesquisa os materiais publicados em periódicos da CAPES, produzidos entre 2007 e 2017.

\subsection{Etapas da Revisão Sistemática}

Uma revisão sistemática consiste na revisão da literatura científica buscando encontrar, avaliar e interpretar as pesquisas mais relevantes de um determinado campo de estudo [Ministério da saúde].

Inicia-se com a definição da questão da pesquisa, fundamental para a determinação da abrangência do estudo e escolha das palavraschave, segue-se com a deliberação dos critérios de elegibilidade, como período de publicação, base de dados, etc. Em seguida é feita a revisão propriamente dita, etapa de leitura dos materiais encontrados, que para este artigo passou por duas etapas: o teste de relevância I, que consistiu 


\section{$16^{\circ}$ \\ ERGODESIGN USIHC CINAHPA}

na leitura dos elementos pré-textuais (títulos e resumos), e o teste de relevância II, que consistiu na leitura na íntegra dos artigos.

A etapa seguinte é a documentação da metodologia, que trata de registrar todas as etapas e critérios adotados, com a finalidade de viabilizar uma revisão da pesquisa de forma clara e transparente. Por último acontece a tabulação e análise dos dados encontrados.

\section{Metodologia}

A pesquisa foi conduzida por meio da pergunta "Como se encontra a produção acadêmica relacionada às avaliações de acessibilidade física dentro das universidades?", e os critérios de inclusão foram: (a) pesquisas que avaliam a acessibilidade física das universidades, (b) artigos publicados no período de 2007 a 2017, (c) artigos em inglês, português ou espanhol, (d) revisados por pares. Por ordem decrescente de relevância, foram selecionados os 100 primeiros artigos relacionados no portal. Os critérios de exclusão foram: (a) artigos que não tinham como foco a avaliação da acessibilidade física de universidades, (b) artigos repetidos, e (c) editoriais, cartas, comentários, revisões, dissertações e teses.

As combinações de palavras-chave escolhidas foram:

- análise AND acessibilidade

- avaliação AND acessibilidade

- acessibilidade AND universidade

- acessibilidade AND "ensino superior"

- avaliação AND acessibilidade AND universidade

- análise AND acessibilidade AND universidade

- avaliação AND acessibilidade AND "ensino superior"

- analysis AND accessibility

- evaluation AND accessibility

- accessibility AND university

- accessibility AND "higher education"

- evaluation AND accessibility AND university $16^{\circ}$ Ergodesign - Congresso Internacional de Ergonomia e Usabilidade de Interfaces Humano Tecnológica: Produto, Informações Ambientes Construídos e Transporte

$16^{\circ}$ USIHC - Congresso Internacional de Ergonomia e Usabilidade de Interfaces Humano Computador

CINAHPA | 2017 - Congresso Internacional de Ambientes Hipermídia para Aprendizagem.

- analysis AND accessibility AND university

- evaluation AND accessibility AND "higher education"

- analysis AND accessibility AND "higher education"

Esta busca levou aos seguintes resultados:

\begin{tabular}{|c|c|}
\hline Termo Pesquisado & $\begin{array}{c}\text { Número de artigos } \\
\text { encontrados }\end{array}$ \\
\hline análise AND acessibilidade & 876 \\
\hline avaliação AND acessibilidade & 669 \\
\hline acessibilidade AND universidade & 824 \\
\hline acessibilidade AND ensino superior & 147 \\
\hline $\begin{array}{l}\text { avaliação AND acessibilidade AND } \\
\text { universidade }\end{array}$ & 574 \\
\hline $\begin{array}{l}\text { análise AND acessibilidade AND } \\
\text { universidade }\end{array}$ & 713 \\
\hline $\begin{array}{l}\text { avaliação AND acessibilidade AND } \\
\text { "ensino superior" }\end{array}$ & 107 \\
\hline $\begin{array}{l}\text { análise AND acessibilidade AND } \\
\text { "ensino superior" }\end{array}$ & 124 \\
\hline analysis AND accessibility & 189.276 \\
\hline evaluation AND accessibility & 82.327 \\
\hline accessibility AND university & 133.088 \\
\hline accessibility AND "higher education" & 11.731 \\
\hline $\begin{array}{l}\text { evaluation AND accessibility AND } \\
\text { university }\end{array}$ & 54.604 \\
\hline $\begin{array}{l}\text { analysis AND accessibility AND } \\
\text { university }\end{array}$ & 115.273 \\
\hline $\begin{array}{l}\text { evaluation AND accessibility AND } \\
\text { "higher education" }\end{array}$ & 5.694 \\
\hline $\begin{array}{l}\text { analysis AND accessibility AND } \\
\text { "higher education" }\end{array}$ & 10.060 \\
\hline
\end{tabular}

Tabela 01 - Artigos encontrados através das combinações de palavras-chave

A aplicação do teste de relevância I (leitura dos elementos pré-textuais e aplicação dos critérios de exclusão) resultou na seleção de 19 artigos, e até este ponto a pesquisa foi conduzida individualmente por cada pesquisadora. Com o resultado do primeiro teste de relevância foi possível desenvolver o Índice de Concordância Kappa (que demonstra a consistência da pesquisa), e entre os pesquisadores esse índice foi de 0,81 (IC $81 \%=0,81-1,00$ ), considerado excelente [OLIVEIRA et al, 2006].

A aplicação do teste de relevância II (leitura na 


\section{$16^{\circ}$ \\ ERGODESIGN \\ USIHC CINAHPA}

íntegra) excluiu mais 8 artigos por não atenderem ao critério de inclusão. Os artigos resultantes foram analisados para identificar como está atualmente a pesquisa sobre acessibilidade física nas universidades.

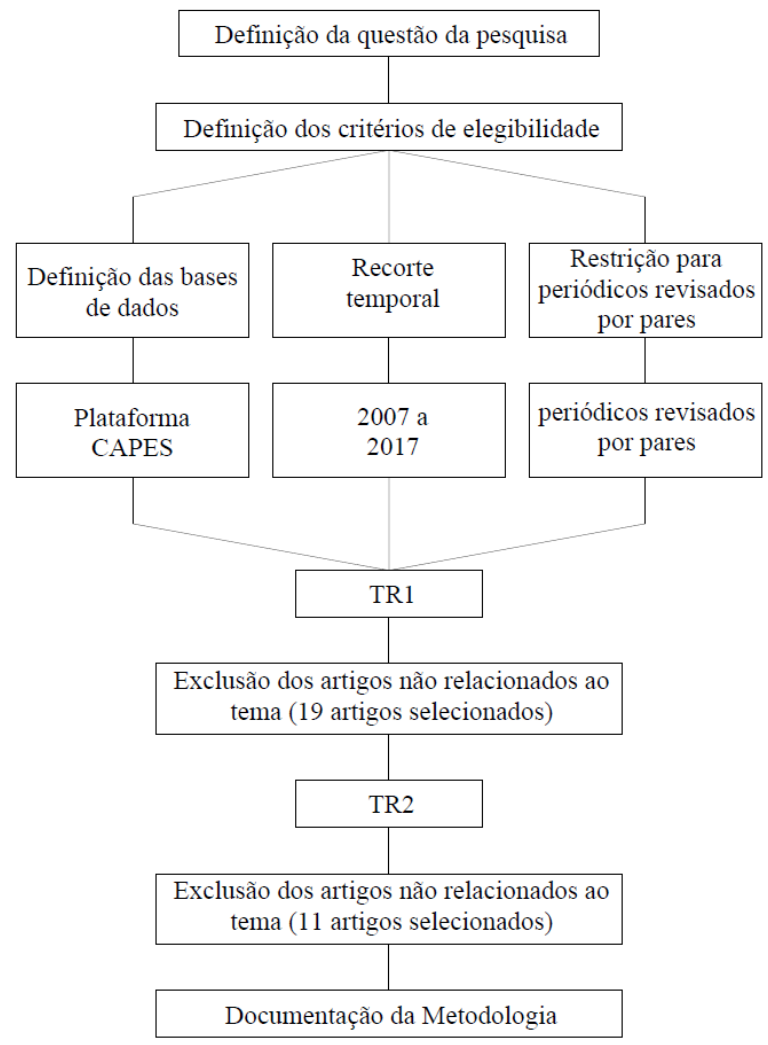

Figura 2: Fluxograma das etapas seguidas para seleção dos artigos.

TR1: Teste de Relevância I, TR2: Teste de Relevância II.

\section{Resultados}

Após o teste de relevância II foram analisados cinco artigos publicados em periódicos internacionais e seis artigos publicados em periódicos nacionais. A análise desses artigos baseou-se nas seguintes perguntas: Qual o foco principal do artigo? Qual o papel da avaliação da acessibilidade física no contexto? Qual a metodologia de avaliação da acessibilidade física utilizada? $16^{\circ}$ Ergodesign - Congresso Internacional de Ergonomia e Usabilidade de Interfaces Humano Tecnológica: Produto, Informações Ambientes Construídos e Transporte

$16^{\circ}$ USIHC - Congresso Internacional de Ergonomia e Usabilidade de Interfaces Humano Computador

CINAHPA | 2017 - Congresso Internacional de Ambientes Hipermídia para Aprendizagem.

Com isso, observaram-se três temáticas principais nas quais os artigos foram categorizados: análise da acessibilidade física propriamente dita, análise da acessibilidade sob a ótica da opinião do usuário e a junção dos dois temas anteriores.

\begin{tabular}{lccc}
\hline \multicolumn{1}{c}{ Tema } & Nacional & Internacional & Total \\
\hline $\begin{array}{l}\text { Acessibilidade } \\
\text { Física }\end{array}$ & 03 & 01 & 04 \\
\hline $\begin{array}{l}\text { Opinião do } \\
\text { usuário }\end{array}$ & 02 & 02 & 04 \\
\hline $\begin{array}{l}\text { Combinação } \\
\text { dos temas }\end{array}$ & 01 & 02 & 03 \\
\hline
\end{tabular}

Tabela 02 - Distribuição temática dos artigos

De todas as publicações em periódicos internacionais, apenas uma tratava exclusivamente da análise física da acessibilidade (e foi desenvolvida por pesquisadores brasileiros, em ambiente universitário brasileiro), as demais focam na opinião do usuário sobre o tema e sobre os ambientes analisados, assim como metade das publicações nacionais.

Também foi interessante encontrar ferramentas tradicionalmente utilizadas em outras áreas de pesquisa sendo utilizadas para este tipo de análise, como a $5 \mathrm{~W} 2 \mathrm{H}$, proposta por Pfitscher [2004] e adaptada para uso em instituições de ensino por Freitas [2013], tradicionalmente utilizada para a elaboração de planos de ação. É importante destacar que a avaliação da infraestrutura tendo como referência as normas e legislação vigente ainda é predominante. [ACIOLY; OLIVEIRA; FREITAS, 2012; AGUIAR; RAMOS; SILVA, 2009; CAVALCANTI; GARCIA; RADOS, 2015]

É evidente a tendência pela busca da opinião do usuário sobre a acessibilidade do ambiente que ocupa. Em todas as pesquisas que trataram do tema foram utilizados questionários tipo "survey", em entrevistas com usuários portadores e não portadores de deficiência [GILSON et al, 2009; SANTOS; MENDONÇA, 


\section{$16^{\circ}$ \\ ERGODESIGN USIHC CINAHPA}

2015; SILVA; MARTINS, 2016; SIMONSON; GLICK; ELLEN C. NOBE, 2013], porém os estudos que combinaram a avaliação do ambiente com a opinião dos usuários trouxeram resultados concretos e muito proveitosos para pesquisadores da área [FREITAS; PFITCHER; BELAN, 2016; LOMBARDI; MURRAY; GERDES, 2011; SUN et al, 2015].

\section{Considerações Finais}

A acessibilidade é um tema pesquisado mundialmente, mesmo quando se busca um contexto mais específico, como o que norteia este artigo. Mesmo num recorte tão específico, podem ser encontradas pesquisas atuais sendo realizadas e publicadas em diversos países e continentes diferentes. A grande tendência observada ao final desta revisão é a inclusão da opinião do usuário como parte da avaliação ou como foco principal do trabalho, principalmente buscando compreender como se relacionam com a ausência ou não da acessibilidade em seu cotidiano.

Nos trabalhos que combinaram a avaliação física e as entrevistas foi encontrado um excelente material, com bastante consistência, e a utilização de ferramentas diferentes do convencional demonstra a versatilidade com que o tema pode ser trabalhado.

Como consequência desta revisão fica a percepção de como a acessibilidade é um tema moderno e ainda em expansão, e que expressa constante necessidade de aprimoramento e ampliação das formas em que é trabalhada e estudada.

\section{Referências Bibliográficas}

\section{ABNT - ASSOCIAÇÃO BRASILEIRA DE NORMAS TÉCNICAS. NBR 9050/2015.}

Acessibilidade a edificações, mobiliário, espaços e equipamentos urbanos. Rio de Janeiro: ABNT, $16^{\circ}$ Ergodesign - Congresso Internacional de Ergonomia e Usabilidade de Interfaces Humano Tecnológica: Produto, Informações Ambientes Construídos e Transporte

$16^{\circ}$ USIHC - Congresso Internacional de Ergonomia e Usabilidade de Interfaces Humano Computador

CINAHPA | 2017 - Congresso Internacional de Ambientes Hipermídia para Aprendizagem.
2015.

\author{
ABNT - ASSOCIAÇÃO BRASILEIRA DE \\ NORMAS TÉCNICAS. NBR 16537/2016. \\ Acessibilidade - Sinalização tátil no piso - \\ Diretrizes para elaboração de projetos e \\ instalação. Rio de Janeiro: ABNT, 2016.
}

ACIOLY, A. S. G.; OLIVEIRA, M. D.; FREITAS, V. H. F. Analysis of accessibility for buildings of a graduation school - An experiment in ergonomics training curriculum. Work, v. 41, n. SUPPL.1, p. 4124-4129, 2012.

AGUIAR, F. D. O.; RAMOS, R. A. R.; SILVA, A. N. R. DA. Cenários de avaliação das restrições à mobilidade dos pedestres em espaços públicos: o caso de um campus universitário. Transportes, v. 17, n. 2, p. 515, 2009.

BRASIL. Decreto n. 5.296, de 02 de dezembro de 2004: Regulamenta as Leis nos 10.048, de 8 de novembro de 2000, que dá prioridade de atendimento às pessoas que especifica, e 10.098, de 19 de dezembro de 2000, que estabelece normas gerais e critérios básicos para a promoção da acessibilidade das pessoas portadoras de deficiência ou com mobilidade reduzida, e dá outras providências.

BRASIL. Decreto n. 5.626, de 22 de dezembro de 2005: Regulamenta a Lei no 10.436, de 24 de abril de 2002, que dispõe sobre a Língua Brasileira de Sinais Libras, e o art. 18 da Lei no 10.098, de 19 de dezembro de 2000.

BRASIL. Lei Federal n. 8.112, de 11 de dezembro de 1990: Dispõe sobre o regime jurídico dos servidores públicos civis da União, das autarquias e das fundações públicas federais. Brasília, 1990.

BRASIL. Lei Federal n. 10.257, de 10 de julho de 2001: Estatuto da Cidade. Brasília,

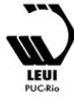


$16^{\circ}$ Ergodesign - Congresso Internacional de Ergonomia e Usabilidade de Interfaces Humano Tecnológica: Produto, Informações Ambientes Construídos e Transporte

$16^{\circ}$ USIHC - Congresso Internacional de Ergonomia e Usabilidade de Interfaces Humano Computador

CINAHPA | 2017 - Congresso Internacional de Ambientes Hipermídia para Aprendizagem.
2001.

BRASIL. Lei Federal n. 10.098, de 19 de dezembro de 2000 - Normas gerais e critérios básicos para a promoção a acessibilidade das pessoas portadoras de deficiência ou com mobilidade reduzida. Brasília, 1999.

CAVALCANTI, C. O. DOS P.; GARCIA, R. I.; RADOS, G. Acessibilidade Física Na Biblioteca Do Instituto Federal De Educação , Ciência E Tecnologia De Santa Catarina, Câmpus Physical Accessibility in the Library of the Federal Institute of Education , Science and Technology of Santa Catarina, Campus Ararangu. Revista ACB:

Biblioteconomia em Santa Catarina, v. 20, p. 483-499, 2015.

FREITAS, K. E. P.; PFITCHER, E. D.; BELAN, A. B. Análise de acessibilidade: aplicação parcial do modelo sicogea em um centro de ensino de uma instituição federal de ensino. Revista de gestão ambiental e sustentabilidade - GeAS, v. 5, p. 120-134, 2016.

GILSON, N. et al. Physical infrastructure and workplace walking in an Australian university campus. Journal of Science and Medicine in Sport, v. 12, n. 1, p. S72, jan. 2009.

GOMES, M. M. A. ; PEREGRINO, Y. ; COURA, P. V. ; SOUZA, R. A. ; Sarmento, Bruna; COSTA, A. D. L. . UFPB Para

Todos: Trilhando Caminhos.... In: III Encontro Nacional de Ergonomia do Ambiente Construído e IV Seminário Brasileiro de Acessibidade Integral, 2011, João Pessoa. Anais do III Encontro Nacional de Ergonomia do Ambiente Construído e IV Seminário Brasileiro de Acessibidade Integral, 2011. p. 1-3.

LEAL, L. N.; THOMÉ, C. Brasil tem 4,6 milhões de deficientes. Disponível em:< http://www.estadao.com.br/noticias/geral,bra sil-tem-45-6-milhoes-dedeficientes,893424>. Último acesso: $24 \mathrm{de}$ março de 2017.

LOMBARDI, A. R.; MURRAY, C.; GERDES, H. College faculty and inclusive instruction: Self-reported attitudes and actions pertaining to Universal Design. Journal of Diversity in Higher Education, v. 4, n. 4, p. 250-261, 2011.

OLIVEIRA, N.S.; OLIVEIRA, J. M.; BERGAMARSCHI, D. P. Concordância entre avaliadores na seleção de artigos em revisões sistemáticas. Revista Brasil epidemiol, v. 9, n. 3, p. 309-315, 2006.

SANTOS, R. A.; MENDONÇA, S. R. D. Universitários cegos: a visão dos alunos e a (falta de visão) dos professores. Revista eCurriculum, v. 13, p. 888-907, 2015.

SASSAKI, R. K. Inclusão: construindo uma sociedade para todos. 8. ed. Rio de Janeiro: WVA, 2010.

SILVA, K. C. DA; MARTINS, S. E. S. DE $O$. Acessibilidade à educação superior brasileira: o que dizem os estudantes com deficiência. Journal of Research in Special Educational Needs, v. 16, p. 116-119, 2016.

SIMONSON, S.; GLICK, S.; ELLEN C. NOBE, M. Accessibility at a public university: student's perceptions. Journal of Facilities Management, v. 11, n. 3, p. 198209, 2013.

SUN, G. et al. Comparing the perception with the reality of walking in a hilly environment: an accessibility method applied to a University campus in Hong Kong. Geospatial health, v. 10, n. 1, p. 340, 2015. 